\title{
Chest trauma: an overview
}

\begin{abstract}
Traumatic damage is the principal cause of death under the age of 45 in the US. Worldwide 5.8 million people die every year from non/intentional traumatic injuries and violence. Chest trauma is a modern major health problem with a high mortality, even in the trauma center hospitals. It is a life-threatening condition, which often involves other anatomical regions that merit simultaneous diagnosis and treatment. The standard management should be multidisciplinary and preferably initiated at the accident site and maintained through patient transportation, during the arrival to emergency room, during transport to surgery, in the operating room and then in ICU. Early diagnosis and initial assertive management are the key to bring down morbidity and mortality. The anesthesiologist has a vital role in the management of these patients throughout the perioperative period.
\end{abstract}

Volume 3 Issue I - 2015

\author{
Víctor Whizar-Lugo,' Alejandra Sauceda- \\ Gastelum,' Adriana Hernández-Armas, ${ }^{2}$ \\ Francisco Garzón-Garnica, ${ }^{3}$ Maribel \\ Granados-Gómez \\ 'Intensive Care Unit, Hospital General de Tijuana, ISESALUD, \\ México \\ ${ }^{2}$ Department of Anesthesia. Hospital General de Tijuana, \\ ISESALUD, México \\ ${ }^{3}$ Anesthesia resident, Universidad La Salle, México D.F \\ ${ }^{4}$ Operating room chief. Hospital Santo Tomás Panamá City, \\ Panamá
}

Correspondence: Víctor Whizar-Lugo, Intensive Care Unit, Hospital General de Tijuana, ISESALUD Av. Centenario I085I, Zona Rio, Tijuana B.C., México, Tel +52664-6848905, Fax +52664-6848906, email vwhizarl@anestesia-dolor.org

Received: September 03, 2015 | Published: September 09, 2015

\section{Introduction}

Acutetrauma fulfills the criteria to classify the disease for a global pandemic, being these frequent source and substantial contribution to morbidity and mortality in the last decades all over the world. However, it has been a significant effort to diminish its impact on humanity. The acute trauma is without a doubt the largest cause of death, taking more lives than HIV, AIDS, tuberculosis, and malaria combined. Acute trauma and injuries due to violence are a growing public health concern which causes 1 out of 10 deaths each year., ${ }^{1,2}$ On a global scaleevery minute nine people die from traumatic injuries; approximately 5.8 million people die every year from nonintentional traumatic injuries and violence. Traffic accidents make up 1.3million, suicides, 844,000 , and homicides 600,000 are the leading cause for these numbers. The majority of these deaths (91\%) occur in underdeveloped countries. Many traumaticlesions require immediate surgical treatment, but in many nondeveloped countries emergency surgery is not readily available due to lack of workforce, medical equipment, and lack of safe anesthesia. ${ }^{3}$ Blunt chest trauma produces $25 \%$ of traumatic deaths; these injuries contribute up to $50 \%$ of global mortality caused by trauma. Blunt chest trauma is commonly associated with multiple organ damage that favor catastrophic patient outcome. A study with 22,613 patients over 16years of age, with blunt thoracic trauma (AIS Chest $\geq 2$ ) pulmonary, cardiac, and vascular lesions were found to be predictors of negative outcome while chest wall lesions -except bilateral flail thorax due to multiple costal fractures- will not influence significantly the mortality rate. Some non-thoracic factors such as; age, blood transfusion, arterial hypotension, and severe lesions were also predictors for the elevated mortality rate. ${ }^{4}$

The studies of Battle et al. ${ }^{5,6}$ with blunt thoracic trauma found that the patients over the age of 65 , who have three or more rib fractures, pneumonia, and pre-existing cardiopulmonary diseases had a higher mortality rate, and the cases with flail thorax had a higher mortality rate which was ISS $\geq 31$, age $\geq 65$ years. In the last grouppulmonary contusion, flail thorax, and prolonged hospitalization were consistently associated with higher mortality rate. Thoracic trauma is responsible for $25 \%$ of all the deaths by trauma; when it is associated with other lesions, produce a fatal outcome in an additional $50 \%$ of polytrauma patients, especially if the cardiovascular system is involved. ${ }^{7,8}$

\section{Type of thorax injuries}

The type of traumatic chest injuries varies widely and essentially depends on the violent environment or the kinematics and severity of the accidents in the diverse geographical regions around the world. The injuries are divided into four groups:
1. Thoracic wall,
2. Lung,
3. Mediastinum, and
4. Diaphragmatic wounds.

\section{Wounds on the thoracic wall}

Rib fractures: Rib fractures are the most common injuries in trauma centers and secondary care hospitals. They are commonly seen in adults, usually secondary to blunt trauma that can be an insignificant injury or rather can lead to serious complications due to injuries of the internal organs. Rib fractures are clinically manifested bylocaltenderness, hematomas, inspiratory pain or while coughing. They tend to be complicated with pneumonia, flail thorax, hemothorax, hemopneumothorax, pulmonary contusions, or vascular lesions. It is mandatory to initiate an effective analgesic plan, being the most proper regional techniques. Byun and $\mathrm{Kim}^{9}$ studied retrospectively 327 patients with an average of 4.8 rib fractures finding flail chest in $22.3 \%$, pulmonary contusion $22 \%$, pneumothorax $31 \%$, hemopneumothorax $20 \%$, hemothorax $62 \%$. $4.3 \%$ where complicated with pneumonia and $95 \%$ of all the patients were treated with antibiotics. The mortality rate was $5.3 \%$. Studies have shown that patients with multiple rib fractures have a decreased quality of life after three years of the initial injury. Only $71 \%$ returns to their regular work $^{10}$ (Figure 1). 


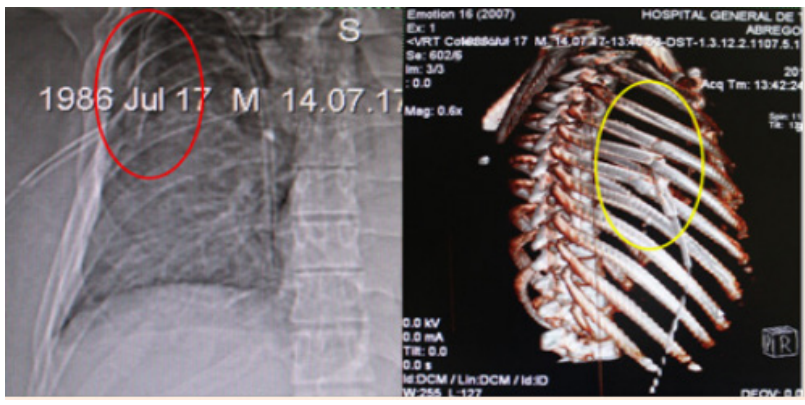

Figure I Rib fractures, pneumothorax and lung contusion.

Sternal fractures: Fractures of the sternum occur with blunt trauma, with a higher frequency in vehicular accidents. Recinos et al. ${ }^{11}$ studied the demographic facts and the outcome of sternum fractures; of 37,087 patients, 125 cases $(0.33 \%)$ had sternum fractures, more commonly in men. The most common injury mechanism was collision between vehicles and patients that have been run over. They were associated with rib fractures and hemothorax/hemopneumothorax. A most recent study ${ }^{12}$ found similar results, with $3.6 \%$ of cardiac contusion, $16.9 \%$ of lumbar fractures. In this study, mortality rate was $7.9 \%$. When this type of fracture is not coupled with another injury, the mortality rate is lower. The clinical diagnosis must be inferred when there is pain and local hypersensitivity. It is confirmed with chest X-ray or with a CT as shown in Figure 2.
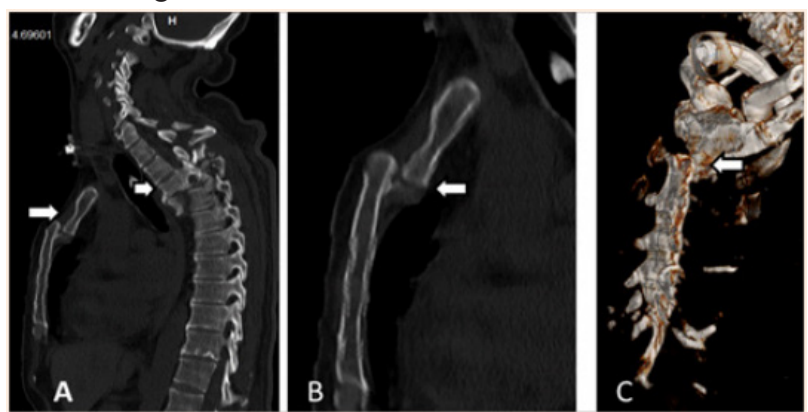

Figure 2 Tomographic image of severe thoracic trauma.A shows a panoramic sagittal section. $B$ and $C$ are close up of the sternal fracture.

Flail chest: The flail chest is an uncommon lesion that usually occurs with a high speed vehicular accident. It is commonly characterized by three or more rib fractures in two or more sites, with or without sternallesion. ${ }^{13}$ where the injured segment of the thoracic wall show paradoxical respiratory movement, mechanical respiratory dysfunction, and frequent respiratory failure. $75 \%$ of the cases are associated with pulmonary contusion, which produces an inflammatory response with right to left shunts, resulting in severe hypoxia, severe pulmonary restriction with the need of tracheal intubation and pulmonary ventilation. Morbidity and mortality are high. The study by Dehghan et al. ${ }^{14}$ with 3,467 cases with flail thorax; of which $75 \%$ were men, $15 \%$ had head injuries, and $54 \%$ had pulmonary contusions. Only $0.07 \%$ had surgical fixation of the thoracic wall and $8 \%$ had epidural analgesia, $44 \%$ were placed a pleural catheter, $21 \%$ required tracheostomy, $59 \%$ required mechanical ventilation, and $82 \%$ were in the ICU for an average of 11.7 days. $21 \%$ of the patients had complications due to pneumonia, adult respiratory distress syndrome in $14 \%$. It is important to mention that only $7 \%$ had sepsis and $16 \%$ died. The treatment is based on analgesia, an aggressive pulmonary hygiene including frequent tracheobronchoscopy, pulmonary physiotherapy especially with hypoxemia chest wall instability means ventilatory problems with trachealintubation or early tracheostomy. ${ }^{15}$
Surgical fixation in costal rib fractures continue to be controversial. Bottlang et al. ${ }^{16}$ used anatomic platesand intramedullary splintsin patients with severe damage level of $28 \pm 10$ with broken ribs $8.5 \pm 2.9$ with good results. These authors have stated that patients reached an $84 \%$ force vital capacity at three monthsand $50 \%$ returned to work at six months. In this trial, there were no deaths. A systematic review and meta-analysis ${ }^{17}$ that includesnine small studies with a total of 538 patients compared the results of non-surgical vs. surgical treatment. The latter treatment was associated with less time on mechanical ventilation support, less pneumonias, less tracheostomies, and a lower mortality rate. A similar study with 753 patients ${ }^{18}$ shared similar results, suggesting that the surgical fixation of rib fractures have significant benefits. This treatment had meaningful results, suggesting that the surgical fixation of the fractured ribs could lead to significant benefits. On the other hand, Cataneo et al. ${ }^{19}$ found evidence in three studies with flail chest patients that non-surgical treatment is better than surgical management. They found fewer cases with pneumonia, thoracic deformities, tracheostomy incidence, mechanical ventilation and their ICU duration (Figure 3).

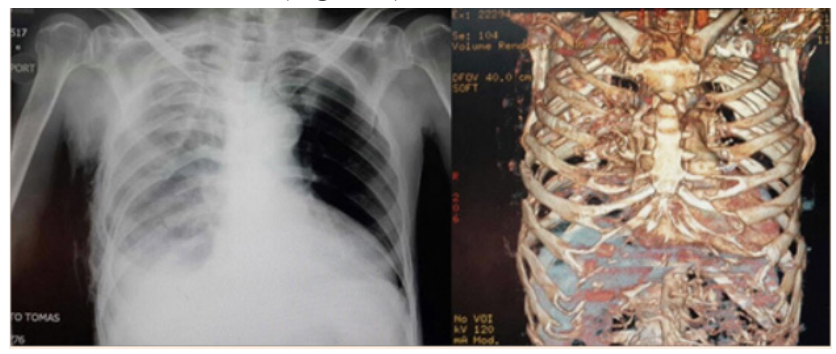

Figure 3 Flail chest and lung contusion.

Pulmonary lesions: This type of lesions can be moderate to severe with imminent death. The clinical skills during pre-hospital care and in the emergency room are essential to determine if there is pulmonary damage as describedin the following paragraphs.

Simple pneumothorax: It refers to a non-complex lesion that contains air within the pleural cavity, non-progressive injury, usually secondary to rib fractures or bronchial ruptures that produces a small pulmonary collapse. Typically is a non-life-threatening damage and most patients are hemodynamically stable, with reduced respiratory sounds, thoracic hyper resonance to percussion, with or without hypoxia. Diagnosis can be corroborated with asimple chest X-ray. Is important to mention that the trachea can be identified in the middle line unlike the tension pneumothorax. Pleural catheter being the standard treatment, however, conservative treatment is another option management in some cases.

Tension pneumothorax: In contrast with previous injury, these patients are in extreme risk of death if a pleural catheter is not inserted immediately. It pertains to a unidirectional valve effect that does not allow the interpleural air outflow which has been accumulating to the point of becoming hypertensive, creating severe collapsed lung, with dangerous contralateral deviation of the mediastinal organs. There is a reduction in venous return. The clinical diagnosis must be immediate, and usually there is no time for a chest X-ray. There are clinical signs of shock, hypoxia, chest hyper resonance and diminish or abolish respiratory murmurs. The affected hemithorax is hyper expanded, with an evident inspiration decrease. This pertains to a super acute and nervousness patient in need of immediate decompression with a thorachostomy needle that transforms a hypertensive pneumothorax in an open pneumothorax. Afterwards, a chest tube is placed and a chest-X-ray is taken to verified diagnosis and proper interpleural tube placement. 
Open pneumothorax: It's the easy entry of air into the pleural cavity through a great chest wall penetrating wound, usually greater than $70 \%$ of the tracheal diameter, as seen in figure 4 . This type of pneumothorax can become hypertensive. There are ventilatory changes and its diagnosis is evident. "Sucking and bubbling" can be seen when air is entering and exiting the pleural space in the site of injury. The diagnosis is confirmed with a plain X-ray and treatment consists of high naso-oral oxygen flow, pleural tube placement and/or a chest wall occlusive wound healing procedure.

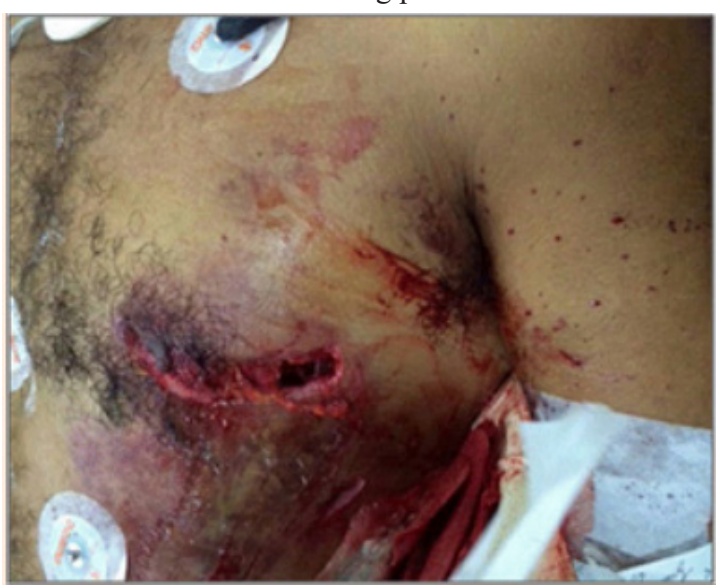

Figure 4 Chest wall stab injury that produced an open pneumothorax.

Hemothorax: It refers to a blood accumulation in the pleural cavity, usually secondary to rib fractures that damage venous vessel, pulmonary trauma and rarely by arterial injury. The clinical diagnosis is similar to that of pneumothorax, it differs in the mate sound at percussive maneuver instead of hyper sonority, respiratory sounds are diminishing or abolish. When hemothorax is massive a mediastinal deviation can be seen. Simple chest X-ray, ultrasound and CT scan studies can confirm the diagnosis. Initial treatment includes high flow oxygen, placement of apleural drainage tube. In stable patients it is recommended to drain up to $1,500 \mathrm{~mL}$ of blood, in the unstable cases only it is allowed to drain up to $1,000 \mathrm{~mL}$ of the blood collected in the pleural cavity. Residual hemothorax may be present in up to $31 \%$, regardless of the position and intrapleural tube length. ${ }^{20}$ It is important to monitor hemodynamic conditions after pleurotomy tube because the blood volume content might have been plugging vascular or pericardial injuries, and the released of this intrapleural pressure can facilitatere-bleeding. The former condition warrants an urgent thorachotomy. ${ }^{21-23}$

Pulmonary contusion: This is a serious, life-threatening injury. It can happen in the absence of rib fracture sorflail chest, especially in young people whodo not have fully ossified ribs. Respiratory failure may develop immediately. It should be suspected in patients with blunt trauma who have $\mathrm{SaO} 2<90 \%, \mathrm{PaO} 2<65$ torr with in the first hour after trauma. Well-defined radiographic pulmonary in filtrates notice able 4 to 6 hours after the traumaare observed. These lesionsare more evidenton CT. Management consists of ventilatory support, restricting intravenous fluids, and antibiotics only when tracheobronchial aspiration is suspected. In patients with flail chest associated with pulmonary contusion management must be more aggressive since their morbidity and mortality is high. In 2012 the Eastern Association for the Surgery of Trauma published their guidelines management in which is recommended fluid restriction at volumes sufficient to maintain adequate tissue perfusion, avoid mechanical ventilation in absence of respiratory failure. Inalert patients requiring mechanical ventilation it is possible to try non-invasive positive pressure ventilation, and when tracheal intubation is required mechanical ventilation must be as short as possible. Pain treatment can be done with regional analgesia with epidural catheter or paravertebral blocks. These guidelines suggest fixing the fractured ribs early in cases of severe thoracic instability. Steroid use is not recommended.

Pulmonaryl aceration: It is an extremely serious injury involving disruption of pulmonary anatomy that is also accompanied by parenchymal hematoma, with or without hemothorax. Suspect in chest radiography and confirmed by CT. These lesions may be come pseudo cysts manifested as hemoptysis due to communicating with the bronchi. When these patients require mechanical ventilation is necessary to avoid high airway pressures and be cautious during alveolar recruitment maneuvers.

Mediastinal lesions: Mediastinal lesions are challenging to diagnosis and management for their extreme gravity. While it may be suspected by the kinematics of trauma, initial clinical evaluation and plain radiographs of the chest are imperative in the diagnosis. Sometimes it requires ultrasound, echocardiography or $\mathrm{CT}$ to confirm the diagnosis, as well as the participation of trauma expert radiologists. All anatomical structures contained in the media stinummay be injured in penetrating or blunt trauma of the chest.

Cardiac lesion: Is a life-threatening injury if the patient is not diagnosed and intervened surgically upon arrival to the hospital. There are three types of injuries of the heart: rupture, contusion and tamponade. Patients with myocardial rupture, usually the right atrium or ventricle, rarely reach the hospital alive. The rupture of the left ventricle is rare and is associated with blunt chest trauma and may be mistaken for aortic injury. ${ }^{24}$ Myocardial contusion is a tolerable injury, manifested by chest pain in only $50 \%$ of cases and may be associated with sterna fracture. There are pleural rub, murmurs and evidence of low cardiac output. Usually there are arrhythmias such as ventricular fibrillation, atrial fibrillation. If the ECG shows ST elevation, it is judicious to perform a coronary angiography. When this injury is suspected CPK and troponin must be requested. If heart failure signs are present it is necessary to use inotropes, intravenous fluids and keep the patient in the intensive care unit. The tamponadeis associated with penetrating trauma of the chest, if the diagnosis is not made immediately most of these patients do not survive their arrival to the emergency room. With only $100 \mathrm{~mL}$ of blood contained in the pericardial sacit is enough to cause heart failure. Beck triad \& Kusmaul $\operatorname{sign}^{25}$ are present in $10 \%$; there is paradoxical pulseand heart failure. Diagnosis must be suspected by simple chest X-ray and confirmed with an ultrasound or echocardiogram. ECG shows low voltage complex and electrical alternans. Treatment must be immediate with pericardiocentesis and sometimes an urgent thoracotomy is needed ${ }^{21,22}$ fluids, amines and respiratory support. CPR is usually ineffective in tamponade cases. In patients with suspected cardiac injury and who are hemodynamically stable, chest CT is recommended, which has high diagnostic significance. ${ }^{26}$ Figures $5 \& 6$ illustrate patients with cardiac lesions resolved with immediate surgery.

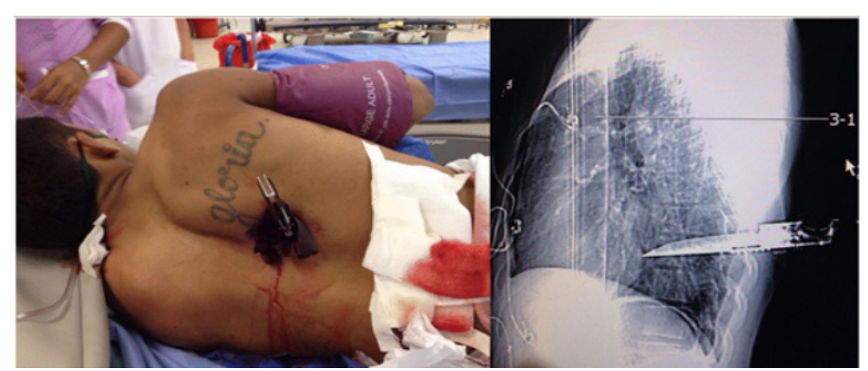

Figure 5 Penetrating thoracic trauma with cardiac lesion. 


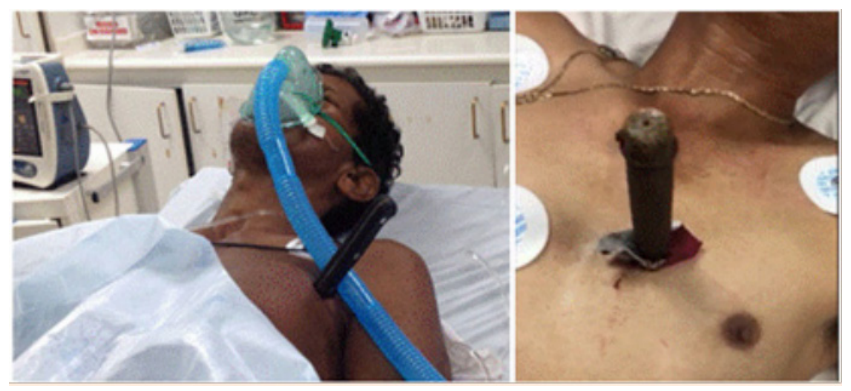

Figure 6 Penetrating thoracic lesions with cardiac involvement.

Pneumopericardium: These injuries are rare and occur after blunt or penetrating trauma. His operative management is not well defined, although it has been mentioned that unstable patients require urgent's urgery. $10 \%$ of these patients may develop hypertension pneumopericardium. ${ }^{27,28}$ The imaging is essential in patients with pericardial injury being ultrasound (FAST) the preferredinitial image study, followed by plain radiography of the chest. Thorax CT is appropriate only in those patients who are hemodynamically stable. ${ }^{29}$

Pneumomediastinum: The incidental pneumomediastinumisa common finding in blunt chest trauma, but their clinical significance is not well defined. It may be associated with esophageal lesions and/ or tracheobronchial injuries that worsen morbidity and mortality. Chouliaras et al. ${ }^{30}$ studied 9,946patients with blunt trauma to the neck and/or chest and found 258 cases $(2.6 \%)$ with pneumomediastinum of which $25 \%$ were diagnosed with plain radiography and $75 \%$ with CT. Of these, $1.6 \%$ had aerodigestive injuries. Lee et al. ${ }^{31}$ retrospectively reviewed 3327 cases of blunt chest trauma who underwent $\mathrm{CT}$, of which $2.2 \%$ were associated with severe pneumomediastinum (ISS and AIS) than patients without pneumomediastinum $(\mathrm{p}<.001)$. They also had higher mortality, longer ICU stay, more days of mechanical ventilation and longer hospital stay. In this studythe size of pneumomediastinum was not associated with mortality, but patients with posterior pneumomediastinum had higher mortality, as when there was air in all mediastinal compartments.Associated hemothorax increased mortality rate to $22.2 \%$.

Vascular injuries: Vascular wounds are rarely seen as most of these patients dieat the trauma scene and the few whoarrive alive at the hospital require rapid diagnosis and immediate surgery. ${ }^{32}$ Timely and complete assessment as well as initial intervention can save these patients. Only $4 \%$ of traumatic chest injuries involve the aorta, innominateve in and artery left carotid, internal mammaryor hilarpulmonary vessels, injuries that can be mildor fatal. The aortic ruptureis a fatal injury that occurs by sudden deceleration in highspeed vehicles, drivers without a seat belt, or penetrating trauma with sudden loss of blood volume that causes immediate death in $90 \%$, and only the remaining $50 \%$ survive in the next 24 hours. The proximal descending aorta is affected more often due to its anatomic structure. When the aortic disruption is not completeit can be seen as mediastinal widening blurring the aorticbutton, fractures ofthe first and second ribs, tracheal deviation to the right, folding down the left main bronchus, deviation of then asogastric tube to the right, and a pical pleuralopacity. Unfortunately all these data are inconsistent. The angio-CT is the cornerstone diagnostic tool, but requires valuable time. Accurate diagnosisis made by angiography. The operative management should be immediately with direct repair, implantation of intravascular graft, including endovascular prosthesis. ${ }^{33,34}$ Patients with fractures of the posterior arch of the first rib must be carefully monitored because of apotentially late aortic injury. Coronary arteries can be involved by direct trauma or secondary to vascular damage. ${ }^{35,36}$
Tracheobronchial lesions: These types of injuries are defined as damage that involves tracheal and/or bronchial cartilage from cricoid cartilage to bronchial division. The majority of these lesions are fatal; however minor cases are well tolerated and often diagnosed in a latter term. Most of the trachea is located in the superior mediastinum space being this a vital reason for a complete assessment in patients with thoracic blunt or penetrating trauma. Is a difficult diagnosis, being the tracheobronchoscopy the standard diagnostic tool; however simple chest X-ray and CT scan may be helpful. This type of lesions may be treated in a conservative form; though surgical treatment reduces intensive care unit stay. ${ }^{37-40}$ Other mediastinal lesions includes esophagic perforation, thoracic duct damage and gas embolism.

Damage to the diaphragm: Diaphragmatic lesions are relatively rare and are associated with high morbidity and mortality. Ninety percent of these injuries occur in blunt trauma related to car accidents. In penetrating injuries, $80 \%$ were secondary to sharp stabbing weapons, with similar percentage involvement in both hemidiaphragms. They can also occur in blunt abdominal trauma without apparent rib injury (Figure 7). Diagnosis is difficult, especially when there is not intrathoracic herniation, and should be suspected from the start. Plain radiography and CT are almost always diagnostic, especially when content intrathoracic abdominal viscera. Surgical management is required as soon as possible, and can be done via laparotomy, thoracotomy, or both. There is often concomitant traumatic injuries. ${ }^{41,42}$ The outcome of these patients is related to the severity of the associated injuries, being the ISS the most important predictor of mortality.

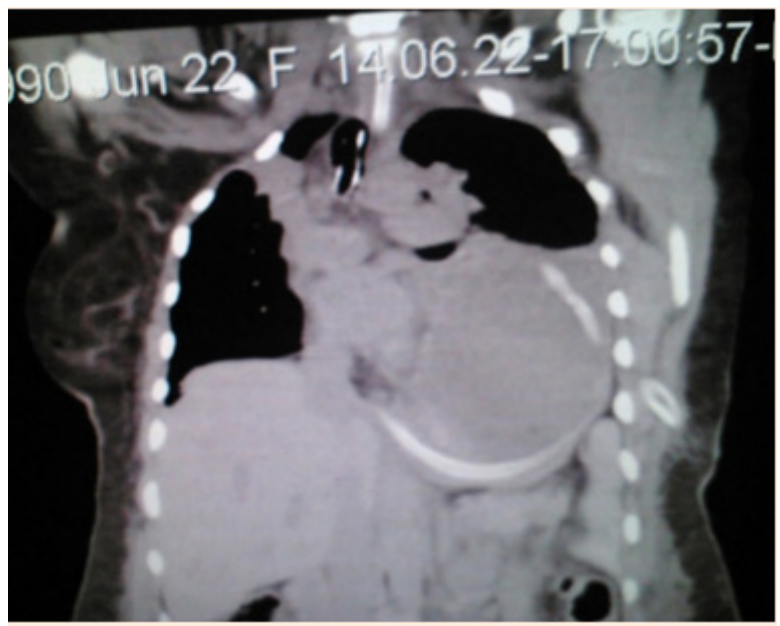

Figure 7 Traumatic trans diaphragmatic hernia showing the stomach in the chest.

\section{Systemic effects of chest trauma}

After the in the initial phase of thoracic trauma there are systemic complications facilitating to rapid evolution like ARDS, ventilatorassociated pneumonia, pulmonary embolism and multiple organ failure. Blunt chest trauma significantly increases the need for ventilatory support and ICU stay time, when compared with non-chest trauma. It has been shown that unilateral lung contusion has a mortality of $25 \%$, when both lungs are contused this mortality rate increased up to $38 \%$, and reaches $50 \%$ if pneumothorax is present. The severity of pulmonary contusion correlates with the incidence of ARDS, especially when more than $20 \%$ has been injured. Pneumonia secondary to chest trauma has an incidence of 5 to $40 \%$, and in some places is the first cause of death. This can be facilitated by hypoventilation induced by pain, atelectasis, and prolonged mechanical ventilation. 
Localimmune mechanisms play an important role in infections, as has been demonstrated both in animals and humans, who injured lungs experience acytokine-mediated inflammatory response which is stronger than the systemic inflammatory response. In experimental animals has been found that the injured lung parenchyma is an important source of proinflammatory mediators that accumulate both in the bronchoalveolar fluid and lung tissue.

While it is difficult to separate the general inflammatory response of thoracictrauma from the local response of the lung damage, it is known thatthe lung parenchyma has a very important role in the inflammatory response and infectious complications. Lung trauma affects gas exchange; early damage of the endothelial membrane increases the thickness of the capillary-alveolar wall which negatively affects gas Exchange with increased intrapulmonary shunt causing hypoxemia, which coupled with the low cardiac output and bleeding anemia, are factors that favor persistence of shock and its consequences, including death. ${ }^{43}$ Experimentally, it has been seen that briefly postrauma chest hiperoxia attenuates the local and systemic inflammatory response, which promotes regeneration of injured tissues, especially associated bone fractures. ${ }^{44,45}$

\section{Diagnosis}

Timely and accurate diagnosis of trauma victims should be in the hands of the most qualified professionals. Unfortunately, this does not happen in most health services, which favors inappropriate treatment, sometimes with catastrophic results. A fuwape et al.. ${ }^{6}$ studied the pattern of missed injuries intrauma at University College Hospital in Ibadan, Nigeria; these authors found in 290 autopsies diagnostic errorsin 55 cases $(18.9 \%)$. There were 79 injuries unnoticed; chest $(43 \%)$ and $26 \%$ abdominal injuries were undiagnosed. Figure 8 shows a general algorithm of the evaluation and management of patients with chest trauma. ${ }^{47}$

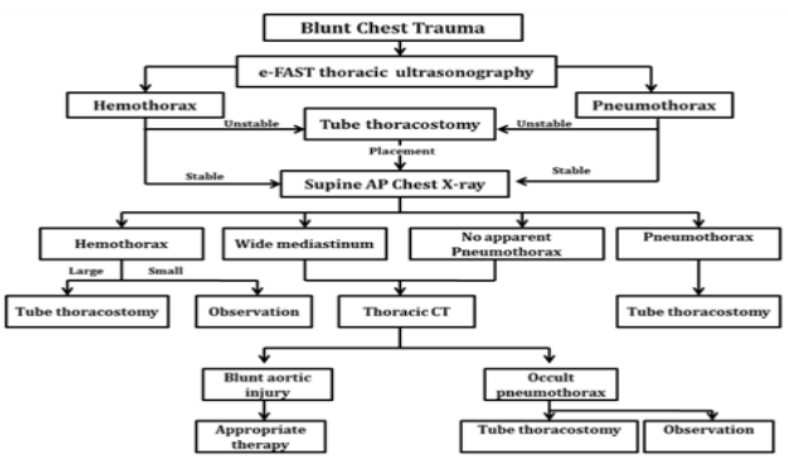

Figure 8 Diagnostic algorithm for chest blunt trauma. e-Fast=Extended focused assessment whit sonography for trauma. AP=Anteroposterior $\mathrm{CT}=$ Computed tomography. ${ }^{47}$

Primary evaluation: In primary assessment of chest trauma patients the process of diagnosis and prompt treatment should be started simultaneously, searching and managing life-threatening lesions such as airway obstruction, tension pneumothorax, open pneumothorax, massive hemothorax, flail chest and cardiac tamponade. It is mandatory to monitor ECG, blood pressure, pulse oximetry and capnography. In this primary evaluation chest X-ray, ultrasound and blood gases are taken and decide whether to intubate the trachea, needle thorachostomy, chest tube thoracotomy in the emergency room, and/or pericardiocentesis.

Secondary assessment: Once the life-threatening lesions are identified and treated a more extend and meticulous evaluation must be done. The first step is to make a complete and detailed review of the case. If there are relatives or friends we must obtain complete data of the patient's medical history that can be relevant for the immediate comprehensive management: diabetes, hypertension, heart disease, lung disease, possible pregnancy, drug addiction, medications, etc. It is also important to collect data on the kinematics of trauma: type of accident or aggression, bleeding at the site, killed, etc. We must review all chest wall, lung parenchyma, diaphragms, mediastinal silhouette and other extra-pulmonary or thoracic injuries. If there is any diagnostic doubt and patient conditions permit, we may request a CT, CT angiography.

Once the diagnosis is definitive, management is required and the patient can be sent home, to hospitalization, to the surgery room, or to the intensive care unit.

\section{Imaging diagnosis of thoracic trauma}

Various imaging techniques are mandatory in the initial diagnosis of thoracic trauma patients. By definition, imaging techniques must accurately diagnose as soon as possible. ${ }^{48}$ Even though echocardiography has an important role in the initial assessment of thoracic trauma patients, plain chest X-ray are the standard of choice in the initial assessment of these patients. Plain chest X-ray are available in almost all emergency rooms, are easily interpreted, reliable, with fast results and affordable. Rib fractures, pneumothorax, pleural effusion, pericardial effusion, blunt pulmonary lesions, and foreign bodies such as knifeor bullets are easily diagnosed. They are also helpful in the initial evaluation of cervicothoracic spine lesion. When plain X-rays are not enough to confirm the clinical diagnosis or there is a reasonable doubt about the radiographic images is recommendable to perform an ultrasound or CT scan. The former one is performed during the secondary assessment. CT scan has a welldefined role in the diagnosis of thoracic trauma; images are more reliable than plain X-ray or ultrasound. ${ }^{48,49} \mathrm{~A}$ recent meta-analysis ${ }^{50}$ include11 studies with a total of 26,371 cases with major trauma, it conclude that a complete body $\mathrm{CT}$ scan is better than conventional $\mathrm{X}$-ray, transabdominal ultrasound or selective CT scan. These researchers found that patients who underwent whole-body CT had lower mortality, less time in the emergency department. There was no effect on the length of stay in ICU or hospital stay. Coincidentally, these patients had longer mechanical ventilation and higher incidence of multiple organ dysfunction/multiple organ failure syndromes. A prospective multicenter study of 5,912 patients $^{51}$ showed that the $\mathrm{CT}$ in patients with severe lesions showed hidden injuries to plain radiography in $71 \%$, of which $37.5 \%$ injuries required surgery. The study by Shannon et al ${ }^{52}$ found that clinical suspicion of traumatic injury correlates somewhat with full body CT; $93 \%$ had fewer injuries than suspected and only $4 \%$ had the same assumed injuries, although $50 \%$ with clinically suspected chest injuries were confirmed by fullbody CT. There were found unexpected lesions in $4 \%$ of which $75 \%$ were considered as severe lesions. Plummer and collaborators ${ }^{53}$ found that transthoracic ultrasound in suspected cardiac injuries did not have a diagnostic impact.

\section{Anesthetic considerations in chest trauma}

The anesthesiologists play an important role in the immediate and mediate treatment of severe trauma victims that complements the services provided by other specialists in the areas of emergency medicine and intensive care. In fact, in some Western European countries anesthesiologists are seen as the "trauma doc". Anesthesiologist in rural areas are involved in the different phases of trauma with higher frequency than their colleagues in metropolitan districts, reason why is of major importance to increase knowledge 
and experience with especial courses in trauma victims primary attention. The contribution of anesthesiologists occurs in different areas or phases according with available resources and/or personal interests and areas of expertise..$^{54,55}$

Pre-surgical evaluation: The assessment must be fast, opportune and at the same time of resuscitation and anesthetic induction. Even after and during anesthesia it is important to obtain more patient data, and overall data about trauma kinetics, transportation and emergency room arrival. If possible, inquire about patient medical history.

Treating shock: The prompt reinstatement of the circulatory volume is critical to restore the supply of oxygen and prevent cell death, especially in vital organs like the brain, heart, liver, kidneys and lungs. While handling the shock usually starts at the site of the accident and in the emergency room, as anesthesiologists we must continue the measures set out above, setting the type and volume used in each

Table I Anesthetics in patients with trauma

\begin{tabular}{ll}
\hline Pharmacological effect & Drug \\
\hline & Ketamine \\
Inductors & Etomidate \\
& Propofol \\
& Thiopental \\
& Fentanyl \\
& Morfine \\
Analgesia & Sufentanil \\
& Vecuronium \\
& Rocuronium \\
& Cisatracurium \\
Muscular Relaxants & Succinylcholine \\
& \\
& \\
Manteinance & Isoflurane, desflurane or sevoflurane
\end{tabular}

TIVA propofol, remifentanil, fentanyl, ketamine, dexmetomidine

\section{ICU treatment considerations}

All patients with moderate or severe chest injuries should be admitted to the ICU, especially when they have been operated or when they require mechanical ventilation. It is necessary to pay special attention to four parameters: intravenous fluids, mechanical ventilation, analgesia and management of concomitant injuries.

Intravenous fluids: The type and volume of intravenous for these patientsis an issue whit an ongoing debate and no consensus have been universally approved. ${ }^{58}$ Therapeutic alternatives are multiple and in patients with chest trauma the main goal is the restoration of circulating volume, shock management, without overloading in order to avoid serious lung inflammatory changes that alter hematosis. It should maintain cerebral, myocardial, lung, liver and kidney perfusion to prevent further damage as already mentioned. Colloids with starches are useful in the initial resuscitation phase and are safe during surgery, but its use has recently been questioned, as they have the possibility of serious side effects, including acute renal failure. Also human albumin and hypertonic solutions have very limited indications in these patients. ${ }^{59-61}$

Ventilatory support: Patients with pulmonary contusion, respiratory failure or abnormalities in blood gases $(\mathrm{pO} 2<60 \mathrm{mmHg}$ and $\mathrm{pCO} 2>$ patient to prevent further damage in the perioperative period, as to adjust the doses of vasoactive drugs, anti-arrhythmic, etc.

Airway management: The patients with trauma must be considered as a difficult airway case, with high possibility of a full stomach and cervical spine injury. The primary goal is to provide adequate oxygenation and prevent further damage. Possible airway obstructions need to be correct, start mask ventilation and tracheal intubation with protective airway maneuvers and rapid sequence..$^{56}$

Choice of anesthetic drugs: There are many different alternatives of anesthetic drugs, but these are chosen in relation to the physical condition of each patient, type of trauma, drug availability and experience of each anesthesiologist. General anesthesia is by far the most used and regional techniques are used for postoperative analgesia. When patients are hemodynamic instable, we must be extra careful with the dosage of inductor agents and maintenance. Table 1 show the most common drug. ${ }^{57}$

$\begin{array}{ll}\text { Dose in } \mathbf{~ m g / k g} & \text { Secondary effects } \\ 0.5-1.0 & \begin{array}{l}\text { Catecholamine increase, } \\ \text { hallucinations, intracranial } \\ \text { hypertension }\end{array} \\ 0.1-0.2 & \text { Adrenal failure, infections } \\ 0.1-1.0 & \text { Cardiac depression, allergies } \\ 0.2-2.0 & \text { Cardiac depression, } \\ 0.001-0.003 & \text { bronchospasm } \\ 0.05-0.1 & \text { Arterial hypotension, } \\ 0.1-0.5 \mu \mathrm{g} / \mathrm{kg} & \text { bradycardia, stiff chest, } \\ 0.15-0.3 & \text { respiratory depression. }\end{array}$

$0.15-0.3$

$0.9-1.2$

0.1

$1.0-1.5$

Dosage depends on hemodynamic stability

$60 \mathrm{mmHg}$ ) require some type of ventilatory support. Pneumothorax should be resolved with a chest tube before starting mechanical ventilation to prevent a secondary tension pneumothorax. When there is a bronco pleural fistula the patient should have a thorachostomy tube to avoid persistence of the fistula. When the fistula is large it is convenient to opt for independent lung ventilation. In cases whit pulmonary contusion it is recommended to use intermittent positive pressure ventilation. Use predetermined volume or pressure mode, low tidal volumes, airways low pressure, with low oxygen concentrationwith the aim to protect the lung. The high PEEP (14-16 $\mathrm{cm} \mathrm{H} 2 \mathrm{O}$ ) is reserved for cases with severe pulmonary compromise. Hypercapnia can be tolerated provided that the $\mathrm{pH}$ exceeds 7.2. There are patients who cantolerate ventilatory non-invasive methods. ${ }^{62}$

Pain treatment: Management of acute traumatic pain and acute postoperative pain in this clinical scenario is mandatory in order to avoid more complications due to the hyper adrenergic state of these patients. Intravenous pure agonist opioids like morphine, fentanyl, and remifentanil are effective in most patients. It is also possible to use opioids patches with fentanyl or buprenorphine for transdermal opioids delivery. This type of administration is non-invasive, effective and safe. It is judicious to combine opioids with non-opioids drugs. Although intravenous opioids are the most appropriate drugs, epidural 
analgesia is associated with a significant outcome improvement in multiple ribs fractures patients. Some peripheral nerve blocks with local anesthetics can be used; intercostal, paravertebral, or interpleural.

\section{Conclusion}

The trauma of the chest is a disease that has worsened due to the modernity that means worldwide increases in violence and accidents. This is a serious condition, which often multifaceted growing involves other anatomical regions that merit simultaneous treatment. Ideal management should be multidisciplinary and ideally start before their arrival at the hospital and maintain it during transport, in the emergency room, the operating room and then in ICU. Early diagnosis and early aggressive management are the key to bring down morbidity and mortality. The anesthesiologist has a vital role in the management of these patients throughout the perioperative period.

\section{Acknowledgements}

We thank anestesia-dolor.org for images 1 to 7 .

\section{Conflicts of interest}

The authors declare that there are no conflicts of interest.

\section{Funding}

None.

\section{References}

1. World Health Organization (WHO). Injuries and violence: the facts. Geneva, Switzerland: World Health Organization; 2010.

2. Lecky FE, Bouamra O, Woodford M, et al. Epidemiology of polytrauma In: Pape HC, et al. (Editors. Damage control management in the polytrauma patient. USA: Springer Sc. LLC; 2010. pp. 13-23.

3. McQueen KA, Hagberg C, McCunn M. The Global trauma burden and anesthesia needs in low and middle income countries. Am Soc Anesth. 2014;78(6):16-19.

4. Huber S, Biberthaler P, Delhey P, et al. Predictors of poor outcomes after significant chest trauma in multiply injured patients:a retrospective analysis from the German Trauma Registry (Trauma Register GU®). Scand J Trauma Resusc Emerg Med. 2014;22(1):52.

5. Battle CE, Hutchings H, Evans PA. Risk factors that predict mortality in patients with blunt chest wall trauma:a systematic review and metaanalysis. Injury. 2014;43(1):8-17.

6. Battle CE, Evans PA. Predictors of mortality in patients with flail chest:a systematic review. Emerg Med. 2015; 204939.

7. LoCicero J, Mattox KL. Epidemiology of chest trauma. Surg Clin North Amer. 1989;69(1):15-19.

8. Fröhlich M, Lefering R, Probst C, et al. Epidemiology and risk factors of multiple-organ failure after multiple trauma:an analysis of 31,154 patients from the Trauma Register DGU. J Trauma Acute Care Surg. 2014;76(4):921-927.

9. Byun JH, Kim HY (2013) Factors affecting pneumonia occurring to patients with multiple rib fractures. Korean J Thorac Cardiovasc Surg. 2013;46(2):130-134.

10. Marasco S, Lee G, Summerhayes R, et al. Quality of life after major trauma with multiple rib fractures. Injury. 2014;46(1):61-65.

11. Recinos G, Inaba K, Dubose J, et al. Epidemiology of sternal fractures. Am Surg. 2009;75(5):401-404.

12. Oyetunji TA, Jackson HT, Obirieze AC, et al. Associated injuries in traumatic sternal fractures:a review of the National Trauma Data Bank. Am Surg. 2013;79(7):702-705.
13. Vana PG, Neubauer DC, Luchette FA. Contemporary management of flail chest. Am Surg. 2014;80(6):527-535.

14. Dehghan N, de Mestral C, McKee MD, et al. Flail chest injuries:a review of outcomes and treatment practices from the National Trauma Data Bank. J Trauma Acute Care Surg. 2014;76(2):462-468.

15. Pettiford BL, Luketich JD, Landreneau RJ. The management of flail chest. Thorac Surg Clin. 2007;17(1):25-33.

16. Bottlang M, Long WB, Phelan D, et al. Surgical stabilization of flail chest injuries with MatrixRIB implants: a prospective observational study. Injury. 2013;44(2):232-238.

17. Leinicke JA, Elmore L, Freeman BD, et al. Operative management of rib fractures in the setting of flail chest: a systematic review and meta-analysis. Ann Surg. 2013;258(6):914-921.

18. Slobogean GP, MacPherson CA, Sun T, et al. Surgical fixation vs non operative management of flail chest: a meta-analysis. $J$ Am Coll Surg. 2013;216(2):302-311.

19. Cataneo AJ, Cataneo DC, de Oliveira FH, et al. Surgical versus nonsurgical interventions for flail chest. Cochrane Data base Syst Rev. 2015;7:CD009919.

20. Kumar S, Agarwal N, Rattan A, et al. Does intrapleural length and position of the intercostal drain affect the frequency of residual hemothorax? A prospective study from north India. J Emerg Trauma Shock. 2014;7(4):274-279.

21. Seamon MJ, Haut ER, Van Arendonk K, et al. An evidence-based approach to patient selection for emergency department thoracotomy:A practice management guideline from the Eastern Association for the Surgery of Trauma. J Trauma Acute Care Surg. 2015;79(1):159-173.

22. Sersar SI, Alanwar MA. Emergency thoracotomies: Two center study. $J$ Emerg Trauma Shock. 2013;6(1):11-15.

23. Simon B, Ebert J, Bokhari F, et al. Management of pulmonary contusion and flail chest: an Eastern Association for the Surgery of Trauma practice management guideline. J Trauma Acute Care Surg. 2012;73(5 Suppl 4):S351-S361.

24. Alameddine AK, Alimov VK, Alvarez C, et al. Unexpected traumatic rupture of left atrium mimicking aortic rupture. J Emerg Trauma Shoc. 2014;7(4):310-312.

25. Asensio JA, Ceballos JJ, Forno W, et al. Lesiones cardiacas penetrantes. Una revisión desde sus orígenes históricos hasta las últimas fronteras en el nuevo milenio. Cirujano General. 2000;22(1):81-91.

26. Plurad DS, Bricker S, Van Natta TL, et al. Penetrating cardiac injury and the significance of chest computed tomography findings. Emerg Radiol. 2013;20(4):279-284.

27. Nicol AJ, Navsaria PH, Hommes M, et al. Management of a pneumopericardium duetopenetrating trauma. Injury. 2014;45(9):1368-1372.

28. Van Peteghem S, Gevaert S. Tension pneumopericardium after blunt chest trauma. Acta Cardiol. 2015;70(1):83.

29. Adams A, Fotiadis N, Chin JY, et al. A pictorial review of traumaticpericardial injuries. Insights Imaging. 2012;3(4):307-311.

30. Chouliaras K, Bench E, Talving P, et al. Pneumomediastinum following blunt trauma: Worth an exhaustive workup? J Trauma Acute Care Surg. 2015;79(2):188-193.

31. Lee WS, Chong VE, Victorino GP (2015) Computed tomographic findings and mortality in patients with pneumomediastinum from blunt trauma. JAMA Surg. 2015;150(8):757-762.

32. Yilmaz TH, Evers T, Sussman M, et al. Operating on penetrating trauma to the mediastinal vessels. Scand J Surg. 2014;103(3):167-174.

33. Mokrane FZ, Revel-Mouroz P, Saint Lebes B, et al. Traumatic injuries of the thoracic aorta: The role of imaging in diagnosis and treatment. Diagnostic and Interventional Imaging. 2015;96(7):693-706. 
34. Park HS, Ryu SM, Cho SJ, Park SM, Lim SH (2014) A treatment case of delayed aortic injury:the patient with posterior rib fracture. Korean J Thorac Cardiovasc Surg. 2014;47(4):406-408.

35. Lim JY, Wolf AS, Flores RM. Thoracic vessel injury. Minerva Chir. 2013;68(3):251-262.

36. Wang H, Jiang L, Zhang L, et al. Compression of the ostium of left main coronary artery caused by aortic root intramural hematoma after blunt thoracic trauma. Int J ClinExp Med. 2015;8(4):6334-6337.

37. Alassal MA, Ibrahim BM, Elsadeck N. Traumatic intrathoracic tracheobronchial injuries: a study of 78 cases. Asian Cardiovasc Thorac Ann. 2014;22(7):816-823.

38. Hwang JJ, Kim YJ, Cho HM, et al. Traumatic tracheobronchial injury:delayed diagnosis and treatment outcome. Korean J Thorac Cardiovasc Surg. 2013;46(3):197-201.

39. Fatimi SH, Hanif HM, Ahmed A, et al. Outcomes of surgical management of tracheobronchial injuries-a case series from a developing country. Chin J Traumatol. 2011;14(3):161-164.

40. Mahmodlou R, Sepehrvand N. Tracheobronchial injury due to blunt chest trauma. Int J Crit Illn Inj Sci. 2015;5(2):116-118.

41. Kadokura M. Traumatic injury of the diaphragm. Kyobu Geka. 2015;68(8):684-688.

42. Hanna WC, Ferri LE. Acute traumatic diaphragmatic injury. Thorac Surg Clin. 2009;19(4):485-489.

43. Neugebauer EA, Waydhas C, Lendemans S, et al. The treatment of patients with severe and multiple traumatic injuries. Dtsch Arztebl Int. 2012;109(6):102-108.

44. Kemmler J, Bindl R, McCook O, et al. Exposure to 100\% oxygen abolishes the impairment of fracture healing after thoracic trauma. PLoS One. 2015;10(7):e0131194.

45. Recknagel S, Bindl R, Brochhausen C, et al. Systemic inflammation induced by a thoracic trauma alters the cellular composition of the early fracture callus. J Trauma Acute Care Surg. 2013;74(2):531-537.

46. Afuwape O, Okolo CA, Ifesanya A. Missed injuries in trauma associated mortalities in a Nigerian teaching hospital. Niger Postgrad Med. 2014;21(1):1-4.

47. Ball CG, Kirkpatrick AW, Feliciano DV. The occult pneumothorax: what have we learned? Can J Surg. 2009;52(5):E173-E179.

48. Palas J, Matos AP, Mascarenhas V, et al. Multi detector computer tomography: evaluation of blunt chest trauma in adults. Radiol Res Pract. 2014;2014:864369.
49. Langdorf MI, Zuabi N, Khan NA, et al. Yield and clinical predictors of thoracic spine injury from chest computed tomography for blunt trauma. West J Emerg Med. 2014;15(4):465-470.

50. Jiang L, Ma Y, Jiang S, et al. Comparison of whole-body computed tomography vs selective radiological imaging on outcomes in major trauma patients: a meta-analysis. Scand J Trauma Resusc Emerg Med. 2014;22(1):54.

51. Langdorf MI, Medak AJ, Hendey GW, et al. Prevalence and clinical import of thoracic injury identified by chest computed tomography but not chest radiography in blunt trauma: Multicenter prospective cohort study. Ann Emerg Med. 2015.

52. Shannon L, Peachey T, Skipper N, et al. Comparison of clinically suspected injuries with injuries detected at whole-body CT in suspected multi-trauma victims. Clin Radiol. 2014.

53. Plummer JM, Condell M, Ferron-Boothe D, et al. The Impact of trans-thoracic ultrasound on cardiac injuries. West Indian Med J. 2014;63(3):258-261.

54. Whizar-Lugo VM. La hora dorada del politraumatizado. Anest Mex. 2004;16(S1):72-76.

55. Vázquez TJ. Anestesia en el paciente con trauma de tórax. Rev Mex Anestesiol. 2007;30(S1):S285-S293.

56. Khan RM, Sharma PK, Kaul N. Airway management after major trauma. Indian J Anaesth. 2011;55(5):463-469.

57. Stene JK, Grande CH M. Anesthesia for trauma patients. In: Longnecker DE, et al. Editors. New York, USA: Anesthesiology; 2008. pp. $1660-1676$.

58. van Haren F, Zacharowski $K$. What's new in volume therapy in the intensive care unit? Best Pract Res Clin Anaesthesiol. 2014;28(3):275-283.

59. Vincent JL, Kellum JA, Shaw A, et al. Should hydroxyethyl starch solutions be totally banned? Crit Care. 2013;17(5):193.

60. Shaw AD, Kellum JA. The risk of AKI in patients treated with intravenous solutions containing hydroxyethyl starch. Clin J Am Soc Nephrol. 2013;8(3):497-503.

61. Schöchl H, Schlimp CJ. Trauma bleeding management: The concept of goal-directed primary care. Anesth Analg. 2014;119:1064-1073.

62. Arora S, Singh PM, Trikha A. Ventilatory strategies in trauma patients. $J$ Emerg Trauma Shock. 2014;7(1):25-31. 